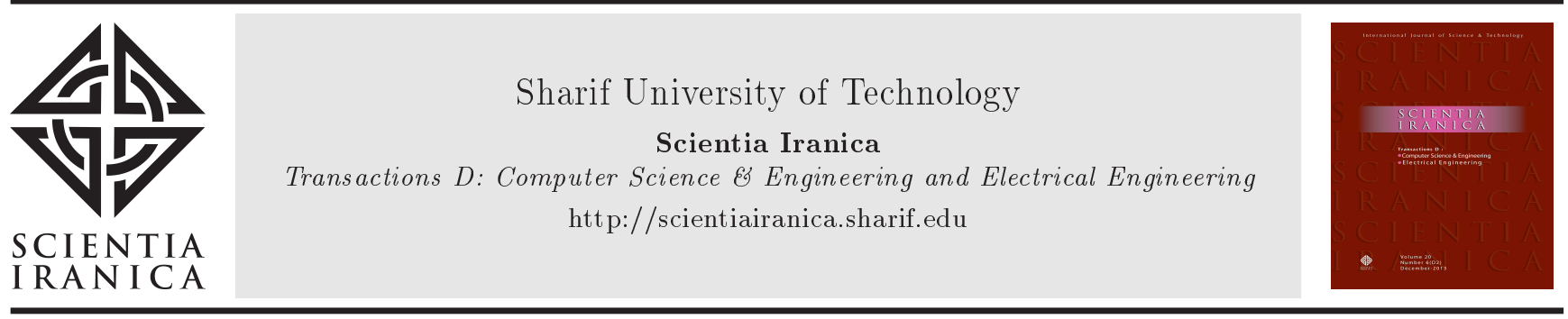

\title{
An efficient observer design method for singular discrete-time systems with time delays and nonlinearity: LMI approach
}

\author{
R. Hajmohammadi and S. Mobayen* \\ Department of Electrical Engineering, University of Zanjan, Zanjan, Iran. \\ Received 28 November 2016; received in revised form 18 March 2017; accepted 19 June 2017
}

\author{
KEYWORDS \\ Singular discrete-time \\ systems; \\ State observer; \\ Time delays; \\ Nonlinearity; \\ Linear matrix \\ inequality.
}

\begin{abstract}
This paper proposes the observer design method for linear and nonlinear singular discrete-time systems with constant time delays. By constructing appropriate Lyapunov-Krasovskii functional and using Linear Matrix Inequality (LMI) technique, the asymptotic convergence criterion is developed in terms of LMIs, which can be solved numerically using MATLAB ${ }^{\circledR}$ LMI ${ }^{\circledR}$ toolbox. The sufficient condition for the existence of a full-order observer is obtained and the states are estimated using Schur complement and S-procedure lemma very well. Moreover, an extension procedure for the observer design of a singular linear system with a time-varying delay is presented. Simulation results are included to prove the efficiency of the suggested approach.
\end{abstract}

(C) 2019 Sharif University of Technology. All rights reserved.

\section{Introduction}

The singular systems are used to describe many practical applications such as robotic systems, biological systems, electrical networks, economic systems, social systems, constrained mechanical systems, space navigation systems, chemical processing, astrophysics, aircraft modelling, etc. [1-6]. Over the past years, the control problem of these classes of systems has been widely studied, and many researches, such as feedback control [7], robust $H_{\infty}$ control [8,9], optimal control [10], impulsive control [11], sliding mode control [12], passive control [13], and fault-tolerant control [14], have been investigated in this field. The control procedure of the singular systems is often based on state-feedback $[15,16]$. However, in some

*. Corresponding author.

E-mail address: mobayen@znu.ac.ir (S. Mobayen) applications, the states of the system are not available for the measurement purpose. For most complex dynamical systems, it is not always feasible to measure all states of the system using a direct measurement procedure [17]. Hence, for the purposes of controller design and dynamic characterization, it is required to design a suitable observer to obtain estimates of the system states, which cannot be measured directly [18]. In this regard, the problem of state observation for the singular systems has undeniable importance. In recent years, many significant works have been investigated in observer design for singular systems.

In [19], the concept of the singular stochastic finite-time boundedness was presented, and finite-time $H_{\infty}$ controller design using observer-based state feedback was addressed for singular Markovian jump systems with time-varying norm-bounded disturbances. The problem of output regulation was considered in [20] for singular systems via dual observer-based compensators where the control purpose can be achieved under weak conditions. The stability analysis and the 
robust dynamic output-feedback control method were proposed in [21] for uncertain continuous-time singular systems with time delays. The design problems of an observer and an observer-based controller based on two LMI conditions were presented in [22] for nonlinear time delay singular systems. In [23], the problem of disturbance-observer-based controller and $H_{\infty}$ control for Markovian jump singular systems with nonlinearities and disturbances was investigated. The problem of observer-based $H_{\infty}$ control was studied in [24] for an uncertain time delay singular system with actuator saturation. Osorio-Gordillo et al. [25] are concerned with the fault-detection and isolation for singular systems via a dynamical observer. In [26], the design method of an observer-based $H_{\infty}$ SMC for the uncertain time delay stochastic singular systems with input nonlinearity was planned. In [27], the design approach to the full-order observer was concerned with time delay singular systems with Lipschitz nonlinearities. Nevertheless, most of the existing researches have been employed in continuous-time singular systems. Design of the state observers for discrete-time singular systems has received little consideration. The observer design problem for discrete-time systems is important due to the prevalence of flexibility and simplicity of design methods for digital systems, as compared to continuous ones. Because it is not tackled in the existing techniques, the proposed work is an important step towards that. In [28], the LMI-based observer design problem was concerned with rectangular discrete-time singular systems with time-varying delays. In [29], the observer-based finite-time $H_{\infty}$ control problem was investigated for discrete-time Markovian jump singular systems with time-varying norm-bounded disturbances. The problem of the observer-based robust controller design was considered in [30] for discretetime singular systems with time-varying delays and norm-bounded uncertainties. The functional observer design was considered in [31] for switched discrete-time singular systems in the presence of unknown inputs, arbitrary switching sequences, and state delays.

In this paper, the observer design is considered for linear and nonlinear singular discrete-time systems with a constant time delay. The proposed observer guarantees asymptotic stability of the singular discretetime systems containing Lipschitz nonlinearities and time delays. Using the Lyapunov-Krasovskii functional, Schur complement, and S-procedure lemmas, the asymptotic stability conditions are established in the form of LMIs and the observer gain, $L$, is determined via LMIs. The main contributions of the proposed method are listed as follows:

(a) Design of a state-observer for the linear and nonlinear singular systems with constant time delays;

(b) Satisfaction of the stability of the overall system with Lipschitz nonlinearity and time delays based on the Lyapunov theory and LMI technique;

(c) Usability of the same approach to continuous-time systems with small changes.

The paper is organized as follows. Section 2 presents the problem formulation of the linear and nonlinear singular discrete-time systems with time delays and introduces two necessary lemmas. Section 3 presents the observer design for the discrete-time linear and nonlinear singular systems and expresses them in the form of LMI formulations. Section 4 gives two simulations to illustrate the validity of our results. Finally, conclusions and future recommendations are drawn in Sections 5 and 6, respectively.

\section{Problem description and assumptions}

\subsection{Singular linear system}

Consider the singular linear discrete-time system with a constant time delay as follows [32]:

$$
\left\{\begin{array}{l}
E x(k+1)=A x(k)+A_{1} x(k-d)+B u(k) \\
y(k)=C x(k)
\end{array}\right.
$$

where $E \in \mathbb{R}^{n \times n}$ is a singular matrix, i.e., $\operatorname{rank}(E)<n$. Vectors $x(k) \in \mathbb{R}^{n}, u(k) \in \mathbb{R}^{p}$, and $y(k) \in \mathbb{R}^{q}$ represent the state, input and output of the system, respectively. Matrices $A, A_{1}, B, C$, and $E$ are known as constant matrices with appropriate dimensions, and $d$ is the nonnegative integer representing the interval time delay.

It is assumed that system (1) is observable, meaning that [32]:

$$
\operatorname{rank}\left[\begin{array}{c}
E \\
C
\end{array}\right]=n
$$

and:

$$
\operatorname{rank}\left[\begin{array}{c}
z E-A \\
C
\end{array}\right]=n, \quad \forall z \in \mathbb{C},
$$

where $|z| \geq 1$ is a finite scalar. Since $\operatorname{rank}\left[\begin{array}{l}E \\ C\end{array}\right]=n$, there exists a full rank matrix $\left[\begin{array}{ll}T & N\end{array}\right]$ such that [33]:

$$
T E+N C=I_{n}
$$

Remark 1. Matrices $T$ and $N$ can be obtained by using the singular value decomposition of matrix $\left[\begin{array}{l}E \\ C\end{array}\right][34,35]$. The general solution for $T$ and $N$ is:

$$
\left[\begin{array}{ll}
T & N
\end{array}\right]=\left[\begin{array}{l}
E \\
C
\end{array}\right]^{\dagger}+Z\left[I_{q+n}-\left[\begin{array}{l}
E \\
C
\end{array}\right]\left[\begin{array}{l}
E \\
C
\end{array}\right]^{\dagger}\right]
$$

where $Z$ is an arbitrary matrix designed, such that $T$ 
is of full rank and $E^{\dagger}$ denotes the pseudo-inverse of matrix $E$.

Lemma 1 (Schur complement) [36-38]: If there exist matrices $S_{1}, S_{2}, S_{3}$ where $S_{1}=S_{1}^{T}, S_{3}=S_{3}^{T}$, then inequality $S_{1}-S_{2} S_{3}^{-1} S_{2}^{T}<0$ is equivalent to:

$$
\left[\begin{array}{ll}
S_{1} & S_{2} \\
S_{2}^{T} & S_{3}
\end{array}\right]<0
$$

\subsection{Singular nonlinear system}

In this section, a class of nonlinear systems with the Lipschitz condition is presented. Consider the singular nonlinear discrete-time system with time delays as follows:

$$
\left\{\begin{array}{l}
E x(k+1)=A x(k)+A_{1} x(k-d)+B u(k)+f(x(k)) \\
y(k)=C x(k)
\end{array}\right.
$$

where nonlinear function $f(x(k)) \in \mathbb{R}^{n}$ is a timevarying vector with $f(0)=0$.

Assumption 1. Nonlinear function $f(x(k))$ is Lipschitz for all $x(k) \in \mathbb{R}^{n}$ and $\hat{x}(k) \in \mathbb{R}^{n}$ which satisfy [3941]:

$$
\|f(x(k))-f(\hat{x}(k))\| \leq \beta\|x(k)-\hat{x}(k)\|,
$$

where $\beta>0$ is a Lipschitz constant. For $\hat{x}(k)=0$, Lipschitz Inequality (7) can be rewritten as follows:

$$
f^{T}(x(k)) f(x(k)) \leq \beta^{2} x^{T}(k) x(k) .
$$

Lemma 2 (S-procedure) [42-44]. Let $Z_{0}, \cdots, Z_{n}$ be symmetric matrices. Consider the following condition on $Z_{0}, \cdots, Z_{n}$ :

$$
\zeta^{T} Z_{0} \zeta>0 \text { for all } \zeta \neq 0
$$

such that:

$$
\zeta^{T} Z_{j} \zeta \geq 0, \quad j=1, \cdots, n .
$$

If there exists non-negative scalars $\tau_{j}$ for $j=1, \cdots, n$, such that $T_{0}-\sum_{j=1}^{n} \tau_{j} T_{j}>0$ holds, then Eq. (9) holds.

\section{Main results}

\subsection{Observer design for a singular linear system}

The control goal is to design an observer by the following form:

$$
\begin{aligned}
\hat{x}(k+1)= & T A \hat{x}(k)+T A_{1} \hat{x}(k-d)+T B u(k) \\
& +L(y(k)-C \hat{x}(k))+N y(k+1),
\end{aligned}
$$

where $\hat{x}(k) \in \mathbb{R}^{n}$ is the estimation of $x(k)$. Matrices
$T$ and $N$ are determined by Eq. (4), and $L$ is a matrix which will be designed such that $\hat{x}(k)$ converges asymptotically to $x(k)$.

Using Eq. (4), dynamics (Eq. (1)) can be written as follows:

$$
\begin{aligned}
x(k+1)= & T A x(k)+T A_{1} x(k-d)+T B u(k) \\
& +N y(k+1) .
\end{aligned}
$$

Our aim is to find a gain matrix $L$ such that the corresponding estimation error is as follows:

$$
e(k)=x(k)-\hat{x}(k),
$$

is asymptotically stable. From Eqs. (10)-(12), the dynamics of the estimation error is given by:

$$
e(k+1)=(T A-L C) e(k)+T A_{1} e(k-d) .
$$

The following theorem gives the necessary and sufficient LMI conditions to design an observer for system (1).

Theorem 1. Consider the singular linear discretetime system (1) and the state observer (10). If there exist matrices $P>0, Q>0$, and $W$ with appropriate dimensions, such that the following LMI is satisfied:

$$
\begin{gathered}
{\left[\begin{array}{cc}
-P+Q & (T A)^{T} P\left(T A_{1}\right)-C^{T} W^{T}\left(T A_{1}\right) \\
* & \left(T A_{1}\right)^{T} P\left(T A_{1}\right)-Q \\
* & * \\
(T A)^{T} P-C^{T} W^{T} \\
0 \\
\\
\quad P
\end{array}\right]<0,}
\end{gathered}
$$

and then by using $L=P^{-1} W$ in Eq. (10), the error dynamics (Eq. (13)) is asymptotically stable.

Proof. Consider the Lyapunov-Krasovskii candidate functional as follows:

$$
V(k)=e^{T}(k) P e(k)+\sum_{i=k-d}^{k-1} e^{T}(i) Q e(i) .
$$

Defining $\Delta V(k)=V(k+1)-V(k)$, we have:

$$
\begin{aligned}
\Delta V(k)= & e^{T}(k+1) P e(k+1)-e^{T}(k) P e(k) \\
& +e^{T}(k) Q e(k)-e^{T}(k-d) Q e(k-d) .
\end{aligned}
$$


Substituting Eq. (13) into Eq. (16) gives:

$$
\begin{aligned}
\Delta V(k)= & e^{T}(k)(T A-L C)^{T} P(T A-L C) e(k) \\
& +e^{T}(k)(T A-L C)^{T} P\left(T A_{1}\right) e(k-d) \\
& +e^{T}(k-d)\left(T A_{1}\right)^{T} P(T A-L C) e(k) \\
& +e^{T}(k-d)\left(T A_{1}\right)^{T} P\left(T A_{1}\right) e(k-d) \\
& -e^{T}(k) P e(k)+e^{T}(k) Q e(k) \\
& -e^{T}(k-d) Q e(k-d) .
\end{aligned}
$$

Eq. (17) can be written as:

$$
\begin{aligned}
& \Delta V(k)=\left[\begin{array}{c}
e(k) \\
e(k-d)
\end{array}\right]^{T} \\
& {\left[(T A-L C)^{T} P(T A-L C)-P+Q\right.} \\
& \left.\begin{array}{l}
(T A-L C)^{T} P\left(T A_{1}\right) \\
\left(T A_{1}\right)^{T} P\left(T A_{1}\right)-Q
\end{array}\right]\left[\begin{array}{c}
e(k) \\
e(k-d)
\end{array}\right] .
\end{aligned}
$$

Inequality $\Delta V(k)<0$ holds if and only if:

$$
\begin{aligned}
& {\left[\begin{array}{c}
(T A-L C)^{T} P(T A-L C)-P+Q \\
*
\end{array}\right.} \\
& (T A-L C)^{T} P\left(T A_{1}\right) \\
& \left.\left(T A_{1}\right)^{T} P\left(T A_{1}\right)-Q\right]<0 .
\end{aligned}
$$

Using Schur complement for Eq. (19) and substituting $W=P L$, LMI (14) is obtained. From Lyapunov stability theory [45], if LMI (14) is satisfied, the error dynamical system (13) is asymptotically stable.

\subsection{Observer design for a singular nonlinear system}

The state-observer for the singular nonlinear discretetime system (6) is designed as follows:

$$
\begin{aligned}
\hat{x}(k+1)= & T A \hat{x}(k)+T A_{1} \hat{x}(k-d)+T B u(k) \\
& +L(y(k)-C \hat{x}(k))+N y(k+1) \\
& +T f(\hat{x}(k)),
\end{aligned}
$$

where matrices $T$ and $N$ are determined by Eq. (4), and $L$ is a matrix which is obtained later, such that $\hat{x}(k)$ asymptotically converges to $x(k)$. The obtaining procedure of the error dynamics is the same as described for singular linear system. Then, the error dynamics for the singular nonlinear system (6) is obtained as follows:

$$
e(k+1)=(T A-L C) e(k)+T A_{1} e(k-d)+\Delta f(x, \hat{x}),
$$

where:

$$
\Delta f(x, \hat{x})=T[f(x(k))-f(\hat{x}(k))] .
$$

The following theorem gives the necessary and sufficient conditions to design the state observer for the singular nonlinear system (6).

Theorem 2. Consider the singular nonlinear system (6) and state observer (20). For a given scalar $\beta>0$, if there exist a scalar $\tau>0$ and matrices $P>0, Q>0$, and $W$ with appropriate dimensions such that the following LMI holds (see Eq. (22)), where $\beta_{1}=\beta \lambda_{\max }(T)$ and $\lambda_{\max }(T)$ denotes the maximum eigenvalue of $T$, and then error dynamical system (21) is asymptotically stable, and matrix $L$ is obtained by $L=P^{-1} W$.

$$
\begin{gathered}
{\left[\begin{array}{cc}
-P+Q+\beta_{1}^{2} \tau & (T A)^{T} P\left(T A_{1}\right)-C^{T} W^{T}\left(T A_{1}\right) \\
* & \left(T A_{1}\right)^{T} P\left(T A_{1}\right)-Q \\
* & * \\
* & * \\
(T A)^{T} P-C^{T} W^{T} & (T A)^{T} P-C^{T} W^{T} \\
\left(T A_{1}\right)^{T} P & 0 \\
P-\tau I & 0 \\
* & -P
\end{array}\right]<0}
\end{gathered}
$$

Proof. Consider the Lyapunov-Krasovskii candidate functional as follows:

$$
V(k)=e^{T}(k) P e(k)+\sum_{i=k-d}^{k-1} e^{T}(i) Q e(i) .
$$

Defining $\Delta V(k)=V(k+1)-V(k)$, we have:

$$
\begin{aligned}
\Delta V(k)= & e^{T}(k+1) P e(k+1)-e^{T}(k) P e(k) \\
& +e^{T}(k) Q e(k)-e^{T}(k-d) Q e(k-d) .
\end{aligned}
$$

Substituting Eq. (21) into Eq. (24) gives:

$$
\begin{aligned}
& \Delta V(k)=e^{T}(k)(T A-L C)^{T} P(T A-L C) e(k) \\
& +e^{T}(k)(T A-L C)^{T} P\left(T A_{1}\right) e(k-d) \\
& +e^{T}(k-d)\left(T A_{1}\right)^{T} P(T A-L C) e(k) \\
& +e^{T}(k-d)\left(T A_{1}\right)^{T} P\left(T A_{1}\right) e(k-d) \\
& +e^{T}(k) P e(k)+e^{T}(k)(T A-L C)^{T} P \Delta f(x, \hat{x}) \\
& +e^{T}(k-d)\left(T A_{1}\right)^{T} P \Delta f(x, \hat{x}) \\
& +\Delta f(x, \hat{x})^{T} P(T A-L C) e(k) \\
& +\Delta f(x, \hat{x})^{T} P\left(T A_{1}\right) e(k-d) \\
& +\Delta f(x, \hat{x})^{T} P \Delta f(x, \hat{x}) \\
& +e^{T}(k) Q e(k)-e^{T}(k-d) Q e(k-d) .
\end{aligned}
$$


Eq. (25) can be written as follows:

$$
\begin{aligned}
& \Delta V(k)=\left[\begin{array}{c}
e(k) \\
e(k-d) \\
\Delta f(x, \hat{x})
\end{array}\right] \\
& {\left[\begin{array}{c}
(T A-L C)^{T} P(T A-L C)-P+Q \\
* \\
*
\end{array}\right.} \\
& \left.\begin{array}{cc}
(T A-L C)^{T} P\left(T A_{1}\right) & (T A-L C)^{T} P \\
\left(T A_{1}\right)^{T} P\left(T A_{1}\right)-Q & \left(T A_{1}\right)^{T} P \\
* & P
\end{array}\right] \\
& {\left[\begin{array}{c}
e(k) \\
e(k-d) \\
\Delta f(x, \hat{x})
\end{array}\right]}
\end{aligned}
$$

Inequality $\Delta V(k)<0$ holds if and only if:

$$
\begin{aligned}
& {\left[\begin{array}{c}
(T A-L C)^{T} P(T A-L C)-P+Q \\
* \\
*
\end{array}\right.} \\
& \left.\begin{array}{cc}
(T A-L C)^{T} P\left(T A_{1}\right) & (T A-L C)^{T} P \\
\left(T A_{1}\right)^{T} P\left(T A_{1}\right)-Q & \left(T A_{1}\right)^{T} P \\
* & P
\end{array}\right]<0 .
\end{aligned}
$$

Since the term $\Delta f(x, \hat{x})$ satisfies the Lipschitz condition, it is shown that:

$$
\|\Delta f(x, \hat{x})\| \leq \beta_{1}\|e(k)\| .
$$

Using Assumption 1, Eq. (28) can be written as follows:

$$
\left[\begin{array}{c}
e(k) \\
e(k-d) \\
\Delta f(x, \hat{x})
\end{array}\right]^{T}\left[\begin{array}{ccc}
\beta_{1}^{2} & 0 & 0 \\
0 & 0 & 0 \\
0 & 0 & -I
\end{array}\right]\left[\begin{array}{c}
e(k) \\
e(k-d) \\
\Delta f(x, \hat{x})
\end{array}\right] \geq 0
$$

Now, by combining Eqs. (26) and (29) and using Lemma 2, inequality $\Delta V(k)<0$ is satisfied if there exists a non-negative scalar $\tau$ such that:

$$
\begin{gathered}
{\left[\begin{array}{cc}
(T A-L C)^{T} P(T A-L C)-P+Q+\beta_{1}^{2} \tau \\
* \\
* & \\
& \\
(T A-L C)^{T} P\left(T A_{1}\right) & (T A-L C)^{T} P \\
\left(T A_{1}\right)^{T} P\left(T A_{1}\right)-Q & \left(T A_{1}\right)^{T} P \\
* & P-\tau I
\end{array}\right]<0 .}
\end{gathered}
$$

Applying Lemma 1 to Eq. (30) and substituting $W=$ $P L$, LMI (22) is obtained. From Eq. (30), it is obvious that $\Delta V(k)<0$ is negative definite; therefore, the error dynamics (21) is asymptotically stable.

\subsection{Extension of observer design for the singular system with time-varying delays}

Consider the singular discrete-time system with timevarying delays as follows:

$$
\left\{\begin{array}{l}
E x(k+1)=A x(k)+A_{1} x(k-d(k))+B u(k) \\
y(k)=C x(k)
\end{array}\right.
$$

where $E \in \mathbb{R}^{n \times n}$ is a singular matrix, i.e., $\operatorname{rank}(E)<n$. Vectors $x(k) \in \mathbb{R}^{n}, u(k) \in \mathbb{R}^{p}$, and $y(k) \in \mathbb{R}^{q}$ represent the state, input and output of the system, respectively. Matrices $A, A_{1}, B, C$, and $E$ are known constant matrices with appropriate dimensions, and $d(k)$ is a time-varying delay which is bounded as follows:

$$
\underline{d} \leq d(k) \leq \bar{d}, \quad(\underline{d}, \bar{d}) \in \mathbb{N},
$$

where $\underline{d}$ and $\bar{d}$ are minimum and maximum delay values, respectively.

The control purpose is to design an observer by the following form:

$$
\begin{aligned}
\hat{x}(k+1)= & T A \hat{x}(k)+T A_{1} \hat{x}(k-d(k))+T B u(k) \\
& +L(y(k)-C \hat{x}(k))+N y(k+1),
\end{aligned}
$$

where $\hat{x}(k) \in \mathbb{R}^{n}$ is the estimation of $x(k)$. Matrices $T$ and $N$ are determined by Eq. (4), and $L$ is a matrix designed, such that $\hat{x}(k)$ converges asymptotically to $x(k)$. Using Eq. (4), the dynamics (31) can be written as follows:

$$
\begin{aligned}
x(k+1)= & T A x(k)+T A_{1} x(k-d(k)) \\
& +T B u(k)+N y(k+1) .
\end{aligned}
$$

Our objective is to find a gain matrix $L$ such that the estimation error (12) is asymptotically stable. Form Eqs. (12), (33), and (34), the dynamics of the estimation error is given by:

$$
e(k+1)=(T A-L C) e(k)+T A_{1} e(k-d(k)) .
$$

The following theorem gives the necessary and sufficient LMI conditions to design observer for singular system (31).

Theorem 3. Consider the singular linear discretetime system (31) and the state observer (33). If there exist matrices $P>0, Q>0$, and $W$ with appropriate dimensions such that the following LMI is satisfied:

$$
\begin{gathered}
{\left[\begin{array}{cc}
-P+(\bar{d}-\underline{d}+1) Q & (T A)^{T} P\left(T A_{1}\right)-C^{T} W^{T}\left(T A_{1}\right) \\
* & \left(T A_{1}\right)^{T} P\left(T A_{1}\right)-Q \\
* & * \\
(T A)^{T} P-C^{T} W^{T} \\
0 \\
-P
\end{array}\right]<0}
\end{gathered}
$$

and then, using $L=P^{-1} W$ in Eq. (33), the error dynamics (35) is asymptotically stable. 
Proof. Consider the Lyapunov-Krasovskii candidate functional as follows:

$$
V(k)=V_{1}(k)+V_{2}(k)+V_{3}(k)
$$

where:

$$
\begin{aligned}
& V_{1}(k)=e^{T}(k) P e(k), \\
& V_{2}(k)=\sum_{i=k-d(k)}^{k-1} e^{T}(i) Q e(i), \\
& V_{3}(k)=\sum_{j=2-\bar{d}}^{1-\underline{d}} \sum_{i=k+j-1}^{k-1} e^{T}(i) Q e(i) .
\end{aligned}
$$

Defining $\Delta V(k)=V(k+1)-V(k)$, we have:

$$
\begin{aligned}
\Delta V_{1}(k)= & e^{T}(k+1) P e(k+1)-e^{T}(k) P e(k), \\
\Delta V_{2}(k)= & e^{T}(k) Q e(k)-e^{T}(k-d(k)) Q e(k-d(k)) \\
& +\sum_{i=k-d(k+1)}^{k-1} e^{T}(i) Q e(i)-\sum_{i=k-d(k)}^{k-1} e^{T}(i) Q e(i), \\
\Delta V_{3}(k)= & (\bar{d}-\underline{d}) e^{T}(k) Q e(k)-\sum_{i=k+1-\bar{d}}^{k-\underline{d}} e^{T}(i) Q e(i) .
\end{aligned}
$$

The third term in Eq. (42) can be written as follows:

$$
\begin{gathered}
\sum_{i=k-d(k+1)}^{k-1} e^{T}(i) Q e(i)=\sum_{i=k+1-\underline{d}}^{k-1} e^{T}(i) Q e(i) \\
+\sum_{i=k+1-d(k+1)}^{k-\underline{d}} e^{T}(i) Q e(i) \\
\leq \sum_{i=k+1-d(k)}^{k-1} e^{T}(i) Q e(i) \\
+\sum_{i=k+1-\bar{d}}^{k-\underline{d}} e^{T}(i) Q e(i) .
\end{gathered}
$$

Using Eq. (44) in Eq. (42), we have:

$$
\begin{aligned}
\Delta V_{2}(k) \leq & e^{T}(k) Q e(k)-e^{T}(k-d(k)) Q e(k-d(k)) \\
& +\sum_{i=k-\bar{d}}^{k-\underline{d}} e^{T}(i) Q e(i) .
\end{aligned}
$$

By taking into account Eqs. (41), (43), and (45), the following equation can be obtained:

$$
\begin{aligned}
\Delta V(k) \leq & e^{T}(k+1) P e(k+1)-e^{T}(k) P e(k) \\
& +(\bar{d}-\underline{d}+1) e^{T}(k) Q e(k) \\
& -e^{T}(k-d(k)) Q e(k-d(k))<0 .
\end{aligned}
$$

Substituting Eq. (35) into Eq. (46) gives:

$$
\begin{aligned}
\Delta V(k) & <e^{T}(k)(T A-L C)^{T} P(T A-L C) e(k) \\
& +e^{T}(k)(T A-L C)^{T} P\left(T A_{1}\right) e(k-d(k)) \\
& +e^{T}(k-d(k))\left(T A_{1}\right)^{T} P(T A-L C) e(k) \\
& +e^{T}(k-d(k))\left(T A_{1}\right)^{T} P\left(T A_{1}\right) e(k-d(k)) \\
& -e^{T}(k) P e(k)+(\bar{d}-\underline{d}+1) e^{T}(k) Q e(k) \\
& -e^{T}(k-d(k)) Q e(k-d(k))<0 .
\end{aligned}
$$

Eq. (47) can be written as follows:

$$
\begin{aligned}
& \Delta V(k)<\left[\begin{array}{c}
e(k) \\
e(k-d(k))
\end{array}\right]^{T} \\
& {\left[\begin{array}{c}
(T A-L C)^{T} P(T A-L C)-P+(\bar{d}-\underline{d}+1) Q \\
*
\end{array}\right.} \\
& \left.\begin{array}{l}
(T A-L C)^{T} P\left(T A_{1}\right) \\
\left.\left(T A_{1}\right)^{T} P\left(T A_{1}\right)-Q\right)
\end{array}\right]\left[\begin{array}{c}
e(k) \\
e(k-d(k))
\end{array}\right]<0 .
\end{aligned}
$$

Inequality $\Delta V(k)<0$ holds if and only if the following inequality is satisfied:

$$
\begin{aligned}
& {\left[(T A-L C)^{T} P(T A-L C)-P+(\bar{d}-\underline{d}+1) Q\right.} \\
& \begin{array}{l}
(T A-L C)^{T} P\left(T A_{1}\right) \\
\left.\left(T A_{1}\right)^{T} P\left(T A_{1}\right)-Q\right]<0 .
\end{array}
\end{aligned}
$$

Using Schur complement for Eq. (49) and substituting $W=P L$, LMI (36) is obtained. From the Lyapunov stability theory, if LMI (36) is satisfied, the error dynamical system (35) is asymptotically stable.

The extension of the observer design for singular nonlinear systems with time-varying delays will be proposed in our future work.

\section{Simulation results}

In this section, two examples are given to demonstrate the efficiency of the proposed method. 
Example 1. Consider the following time delay discrete-time singular system:

$$
\begin{aligned}
& {\left[\begin{array}{ll}
1 & 2 \\
1 & 2
\end{array}\right] } {\left[\begin{array}{l}
x_{1}(k+1) \\
x_{2}(k+1)
\end{array}\right] } \\
&=\left[\begin{array}{cc}
-0.5 & 0 \\
0 & 0.4
\end{array}\right]\left[\begin{array}{l}
x_{1}(k) \\
x_{2}(k)
\end{array}\right] \\
&+\left[\begin{array}{cc}
0.1 & 0.1 \\
0.3 & 0
\end{array}\right]\left[\begin{array}{l}
x_{1}(k-1) \\
x_{2}(k-1)
\end{array}\right]+\left[\begin{array}{l}
1 \\
0
\end{array}\right] u(k), \\
& y(k)=\left[\begin{array}{ll}
1 & 0
\end{array}\right]\left[\begin{array}{l}
x_{1}(k) \\
x_{2}(k)
\end{array}\right] .
\end{aligned}
$$

For the simulation purpose, the initial conditions are set as follows: $x_{1}(0)=-0.5, x_{2}(0)=0.4, \hat{x}_{1}(0)=0.3$, and $\hat{x}_{2}(0)=-1.2$. The constant time delay is given as $d=1$ second. Matrices $T$ and $N$ are calculated from Eq. (4) as follows:

$$
\begin{aligned}
& T=\left[\begin{array}{ll}
-1 & 1 \\
0.5 & 0
\end{array}\right], \\
& N=\left[\begin{array}{c}
1 \\
-0.5
\end{array}\right] .
\end{aligned}
$$

Using Theorem 1, observer gain $L$ is obtained as follows:

$$
L=\left[\begin{array}{c}
0.5601 \\
-0.2470
\end{array}\right]
$$

Figures 1 and 2 show the states of the system and their estimates. As seen from these figures, the states of the observer converge to the states, appropriately.

Example 2. Consider the following nonlinear time delay discrete-time singular system:

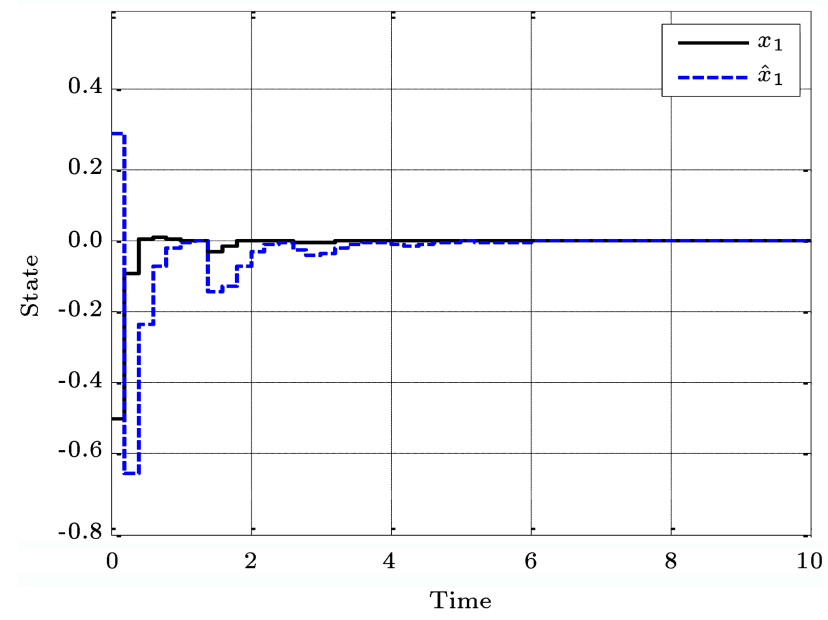

Figure 1. State $x_{1}$ and its estimate $\hat{x}_{1}$.

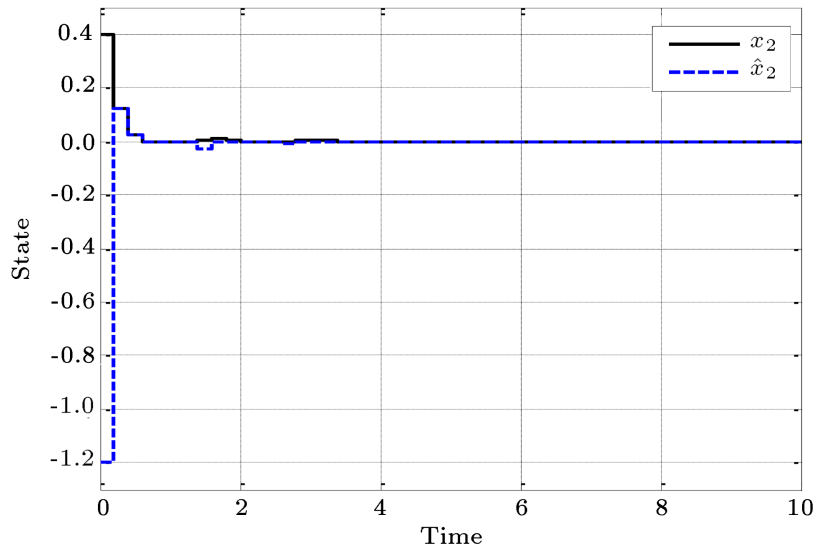

Figure 2. State $x_{2}$ and its estimate $\hat{x}_{2}$.

$$
\begin{aligned}
{\left[\begin{array}{lll}
1 & 2 & 1 \\
0 & 2 & 1 \\
1 & 0 & 0
\end{array}\right] } & {\left[\begin{array}{c}
x_{1}(k+1) \\
\left.x_{2}(k+1)\right) \\
x_{3}(k+1)
\end{array}\right] } \\
= & {\left[\begin{array}{ccc}
0.153 & 0.045 & 0.069 \\
0.156 & 0.252 & 0.156 \\
0.135 & -0.171 & -0.636
\end{array}\right]\left[\begin{array}{l}
x_{1}(k) \\
x_{2}(k)
\end{array}\right] } \\
& +\left[\begin{array}{ccc}
-0.2 & -0.2 & 0 \\
0.6 & -0.5 & 0 \\
-0.2 & 0.2 & 0.7
\end{array}\right]\left[\begin{array}{l}
x_{1}(k-0.2) \\
x_{2}(k-0.2) \\
x_{3}(k-0.2)
\end{array}\right] \\
& +\left[\begin{array}{c}
1 \\
1 \\
0.2
\end{array}\right] u(k)+\left[\begin{array}{c}
0 \\
0.05 \sin \left(x_{3}(k)\right) \\
0
\end{array}\right],
\end{aligned}
$$

$$
y(k)=\left[\begin{array}{lll}
1 & 0 & 1
\end{array}\right]\left[\begin{array}{l}
x_{1}(k) \\
x_{2}(k) \\
x_{3}(k)
\end{array}\right] \text {. }
$$

For the simulation purpose, the initial conditions are set as follows: $x_{1}(0)=0.5, x_{2}(0)=-0.6, x_{3}(0)=$ $0.4, \hat{x}_{1}(0)=0.47, \hat{x}_{2}(0)=-0.58$, and $\hat{x}_{3}(0)=0.46$. The constant time delay is specified as $d=0.2$ second. The Lipschitz value is given by 0.01 . Matrices $T$ and $N$ are obtained by Eq. (4) as follows:

$$
T=\left[\begin{array}{ccc}
1 & -1 & 0 \\
0.5 & 0 & 0 \\
0 & 0 & -1
\end{array}\right], \quad N=\left[\begin{array}{c}
0 \\
0 \\
-0.5
\end{array}\right]
$$

The gain of the observer is attained using Theorem 2 as follows:

$$
L=\left[\begin{array}{c}
-0.1002 \\
0.4053 \\
0.8253
\end{array}\right] \text {. }
$$

Figures 3-5 show the states of the system and their estimates. As observed in these figures, the states of the observer system can converge to those of the actual system as well. 


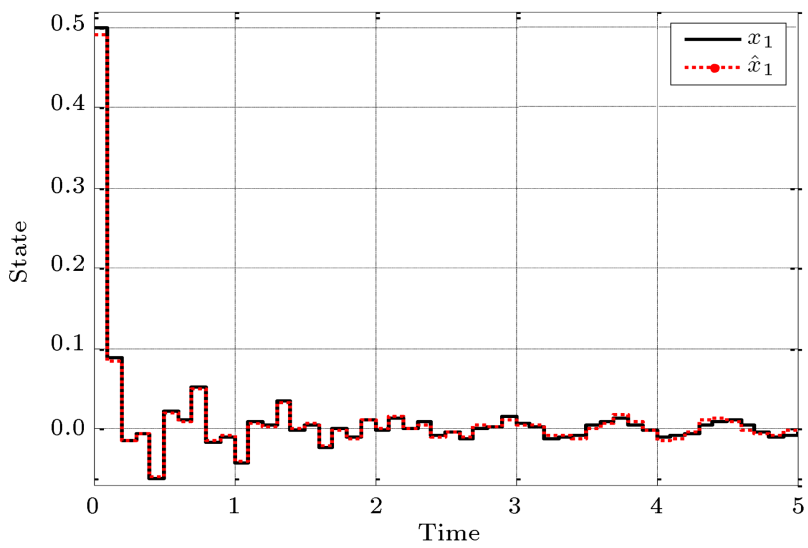

Figure 3. State $x_{1}$ and its estimate $\hat{x}_{1}$.

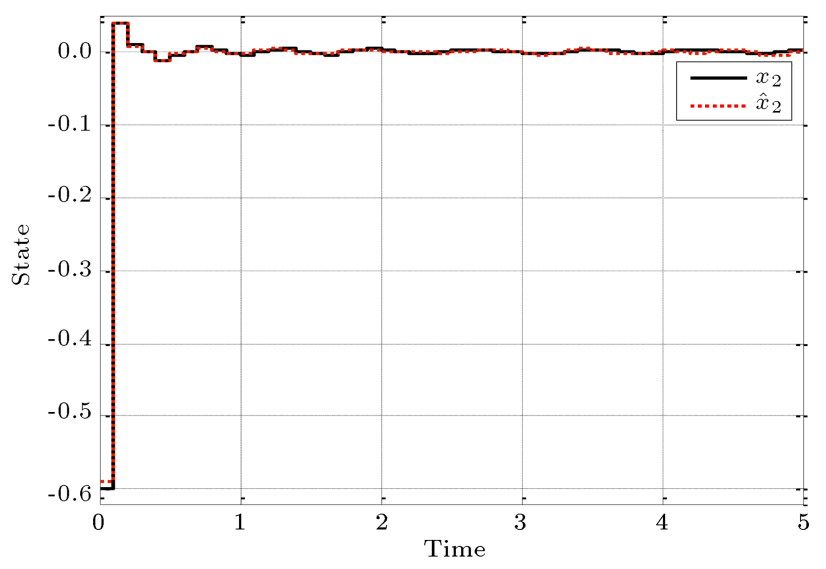

Figure 4. State $x_{2}$ and its estimate $\hat{x}_{2}$.

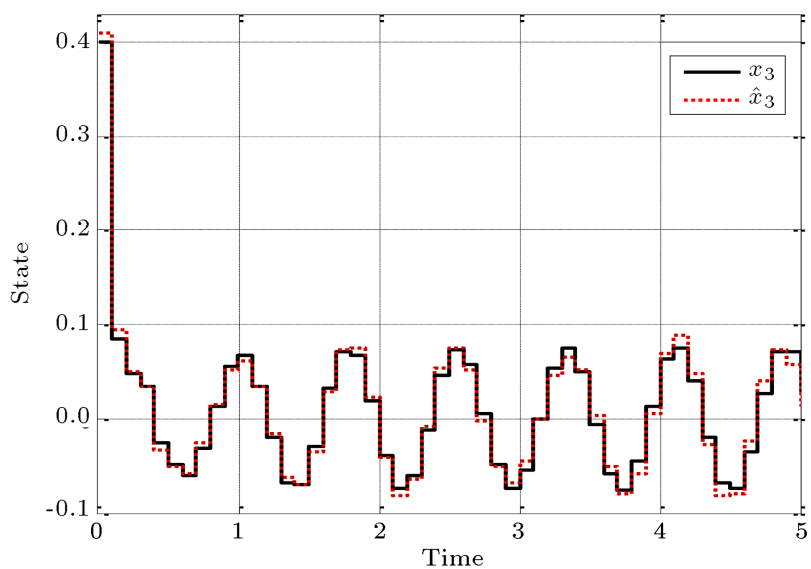

Figure 5. State $x_{3}$ and its estimate $\hat{x}_{3}$.

\section{Conclusions}

This paper investigated the design problem of the state observers for linear and nonlinear singular discretetime systems with constant time delays. Using Schur complement and S-procedure lemma, the necessary and sufficient conditions were given, such that the designed observers estimated the states of the systems. By constructing suitable Lyapunov-Krasovskii functional, the asymptotic convergence criteria were provided in the form of LMIs. Furthermore, the procedure of the observer design was extended for singular linear system with a time-varying delay. Finally, two numerical examples were provided to illustrate the accuracy and efficiency of the proposed method.

\section{Future recommendation}

This approach can be considered as a promising way to implement the practical control problems of singular discrete-time systems; with a little change, it can be applied to continuous systems. The extension of the observer design for singular linear and nonlinear systems with multiple time-varying delays will be presented in the future work.

\section{Acknowledgements}

The authors would like to thank editor, associate editor and the anonymous reviewers for their much time and constructive comments to review and improve this paper. Moreover, the authors would like to appreciate Prof. Fairouz Tchier for her useful suggestions.

\section{References}

1. Zhai, D., Zhang, Q.L., and Li, J.H. "Fault detection for singular multiple time-delay systems with application to electrical circuit", J. Franklin Inst., 351, pp. 54115436 (2014).

2. Shengyuan, X., Dooren-Paul, V., Radu, S., and Lam, J. "Robust stability and stabilization for singular systems with state delay and parameter uncertainty", IEEE Trans. Automat. Control, 47, pp. 1122-1128 (2002).

3. Lin, J.L., and Chen, S.J. "Robustness analysis of uncertain linear singular systems with output feedback control", IEEE Trans. Automat. Control, 40, pp. 19241929 (1999).

4. Zhao, F., Zhang, Q., and Zhang, Y. " $H_{\infty}$ filtering for a class of singular biological systems", IET Control Theory Appl., 9, pp. 2047-2055 (2015).

5. Zerrougui, M., Darouach, M., Boutat-Baddas, L., and Ali, H.S. " $H_{\infty}$ filtering for singular bilinear systems with application to a single-link flexible-joint robot", Int. J. Control Autom. Syst., 12, pp. 590-598 (2014).

6. Balaji, S. "A new Bernoulli wavelet operational matrix of derivative method for the solution of nonlinear singular Lane-Emden type equations arising in astrophysics", J. Comput. Nonlinear Dynam., 11(5), 051013-11 (2016).

7. Lin, D., Lan, W., and Li, M. "Composite nonlinear feedback control for linear singular systems with input saturation", Syst. Control Lett., 60, pp. 825-831 (2011). 
8. Ma, S., Zhang, C., and Cheng, Z. "Delay-dependent robust $H_{\infty}$ control for uncertain discrete-time singular systems with time-delays", J. Comput. Appl. Math., $\mathbf{2 1 7}(1)$, pp. 194-211 (2008).

9. Khandani, K., Majd, V.J., and Tahmasebi, M. "Integral sliding mode control for robust stabilisation of uncertain stochastic time-delay systems driven by fractional Brownian motion", Int. J. Syst. Sci., 48(4), pp. $828-837$ (2017).

10. Mohan, B.M. and Kar, S.K. "Optimal control of singular systems via orthogonal functions", Int. $J$. Control Autom. Syst., 9(1), pp. 145-150 (2011).

11. Shi, S., Zhang, Q., Yuan, Z., and Liu, W. "Hybrid impulsive control for switched singular systems", IET Control Theory Appl., 5(1), pp. 103-111 (2011).

12. Li, J.N., Su, H., Zhang, Y., Wu, Z.G., and Chu, J. "Chattering free sliding mode control for uncertain discrete time-delay singular systems", Asian J. Control, 15(1), pp. 260-269 (2013).

13. Li, Q., Zhang, Q., Yi, N., and Yuan, Y. "Robust passive control for uncertain time-delay singular systems", IEEE Trans. Circuit. Syst. I Reg. Paper, 56(3), pp. 653-663 (2009).

14. Zuo, Z., Ho, D.W., and Wang, Y. "Fault tolerant control for singular systems with actuator saturation and nonlinear perturbation", Automatica, 46(3), pp. 569-576 (2010).

15. Feng, Y. and Yagoubi, M. "On state feedback $H_{\infty}$ control for discrete-time singular systems", IEEE Trans. Automat. Control, 58(10), pp. 2674-2679 (2013).

16. Ma, Y. and Fu, L. " $H_{\infty}$ robust exponential stability and memory state feedback control for uncertain singular time-delay systems with saturating actuators", IET Control Theory Appl., 10(3), pp. 328-338 (2016).

17. Liu, P., Song, H., and Li, X. "Observe-based projective synchronization of chaotic complex modified Van Der Pol-Duffing oscillator with application to secure communication", J. Comput. Nonlinear Dynam., 10(5), 051015-7 (2015).

18. Zhang, Y. and Sinha, S.C. "Observer design for nonlinear systems with time-periodic coefficients via normal form theory", J. Comput. Nonlinear Dynam., 4(3), 031001-10 (2009).

19. Zhang, Y., Cheng, W., Mu, X., and Guo, X. "Observer-based finite-time control of singular Markovian jump systems", J. Appl. Math., 2012, 19 pages (2012).

20. Deutscher, J. "Output regulation for linear singular systems using dual-observer based compensators", Int. J. Control, 86(5), pp. 934-941 (2013).

21. Kchaou, M., Gassara, H., and El-Hajjaji, A. "Robust observer-based control design for uncertain singular systems with time-delay", Int. J. Adapt. Control Signal Process., 28(2), pp. 169-183 (2014).

22. Hassan, L., Zemouche, A., and Boutayeb, M. "Robust observer and observer-based controller for time-delay singular systems", Asian J. Control, 16(1), pp. 80-94 (2014).

23. Yao, X., Zhu, L., and Guo, L. "Disturbance-observerbased control \& $H_{\infty}$ control for non-linear Markovian jump singular systems with multiple disturbances", IET Control Theory Appl., 8(16), pp. 1689-1697 (2014).

24. Ma, Y. and Yan, Y. "Observer-based $H_{\infty}$ control for uncertain singular time-delay systems with actuator saturation", Optim. Control Appl. Method., 37(5), pp. 867-884 (2016).

25. Osorio-Gordillo, G.L., Darouach, M., Boutat-Baddas, L., and Astorga-Zaragoza, C.M. "Dynamical observerbased fault detection and isolation for linear singular systems", Syst. Sci. Control Eng., 3(1), pp. 189-197 (2015).

26. Kao, Y., Xie, J., Wang, C., and Karimi, H.R. "Observer-based $H_{\infty}$ sliding mode controller design for uncertain stochastic singular time-delay systems", Circuit. Syst. Signal Process., 35(1), pp. 63-77 (2016).

27. Nguyen, M.C., Trinh, H., and Nam, P.T. "Nonlinear observer design for a class of singular timedelay systems with Lipschitz non-linearities", IMA $J$. Math. Control Inform, 34(3), pp. 919-935 (2017). DOI: 10.1093/imamci/dnw006

28. Lin, Y. "Observer design for rectangular discrete-time singular systems with time-varying delay", Int. $J$. Phys. Sci., 7(3), pp. 423-431 (2012).

29. Zhang, Y., Shi, P., and Nguang, S.K. "Observer-based finite-time $H_{\infty}$ control for discrete singular stochastic systems", Appl. Math. Lett., 38, pp. 115-121 (2014).

30. Kchaou, M., Tadeo, F., Chaabane, M., and Toumi, A. "Delay-dependent robust observer-based control for discrete-time uncertain singular systems with interval time-varying state delay", Int. J. Control Automat. Syst., 12(1), pp. 12-22 (2014).

31. Lin, J., Shi, Y., Gao, Z., and Ding, J. "Functional observer for switched discrete-time singular systems with time-delays and unknown inputs", IET Control Theory Appl., 9(14), pp. 2146-2156 (2015).

32. Dai, L. "Singular control systems", Lecture Notes in Control and Information Sciences, Springer (1989).

33. Wang, Z., Shen, Y., Zhang, X., and Wang, Q. "Observer design for discrete-time descriptor systems: An LMI approach", Syst. Control Lett., 61, pp. 683-687 (2012).

34. Darouach, M. and Boutayeb, M. "Design of observers for descriptor systems", IEEE Trans. Autom. Control, 40, pp. 1323-1327 (1995).

35. Koenig, D. "Observer design for unknown input nonlinear descriptor systems via convex optimization", IEEE Trans. Autom. Control, 51, pp. 1047-1052 (2006).

36. Scherer, C. and Weiland, S. "Linear matrix inequalities in control", Lecture Notes, Delft University, The Netherlands (2005). 
37. Mobayen, S. and Tchier, F. "An LMI approach to adaptive robust tracker design for uncertain nonlinear systems with time-delays and input nonlinearities", Nonlinear Dyn., 85(3), pp. 1965-1978 (2016).

38. Mobayen, S. and Tchier, F. "A new LMI-based robust finite-time sliding mode control strategy for a class of uncertain nonlinear systems", Kybernetika, 51(6), pp. 1035-1048 (2015).

39. Rehan, M., Hong, K.S., and Ge, S.S. "Stabilization and tracking control for a class of nonlinear systems", Nonlinear Anal. Real World Appl., 12, pp. 1786-1796 (2011).

40. Wang, D., Song, F., and Zhang, W. "State estimation for discrete-time systems with generalized Lipschitz nonlinear dynamics", Adv. Differ. Eq., 2015(1), p. 307 (2015).

41. Mobayen, S. and Tchier, F. "Synchronization of a class of uncertain chaotic systems with Lipschitz nonlinearities using state-Feedback control design: a matrix inequality approach", Asian J. Control, 20(1), pp. 7185 (2018).

42. Wu, H.N. "Delay-dependent stability analysis and stabilization for discrete-time fuzzy systems with state delays: a fuzzy Lyapunov-Krsovskii functional approach", IEEE Trans. Syst. Man Cybern. B Cybern., 36(4), pp. 954-962 (2006).

43. Mobayen, S. "Optimal LMI-based state feedback stabilizer for uncertain nonlinear systems with time-varying uncertainties and disturbances", Complexity, 21(6), pp. 356-362 (2016).

44. Mobayen, S. "An LMI-based robust tracker for uncertain linear systems with multiple time-varying de- lays using optimal composite nonlinear feedback technique", Nonlinear Dyn., 80(1-2), pp. 927-917 (2015).

45. Khalil, H.K. and Grizzle, J.W., Nonlinear Systems, New Jersey: Prentice Hall (1996).

\section{Biographies}

Rahman Hajmohammadi received the MSc degree in Control Engineering from the Department of Electrical Engineering, Shahid Bahonar University of Kerman, Kerman, Iran, in 2011. He focuses on development and implementation of new nonlinear control methods on robotic systems. His research interests include nonlinear control, robotic systems, adaptive control, optimal control, and LMIs.

Saleh Mobayen was born in Khoy, Iran, in December 1983. He received the BS and MSc degrees in Control Engineering from the University of Tabriz, Tabriz, Iran in 2007 and 2009, respectively, and received his $\mathrm{PhD}$ in Control Engineering from Tarbiat Modares University, Tehran, Iran in 2012. Since 2013, he has been as an Assistant Professor and a faculty member with the Department of Electrical Engineering of University of Zanjan, Zanjan, Iran. He has published several papers in the national and international journals. He is a member of IEEE and serves as a member of program committee of several international conferences. His research interests include control theory, sliding mode control, robust tracking, non-holonomic robots, and chaotic systems. 